\title{
Determination of the Allelopathic Potential of Cambodia's Medicinal Plants Using the Dish Pack Method
}

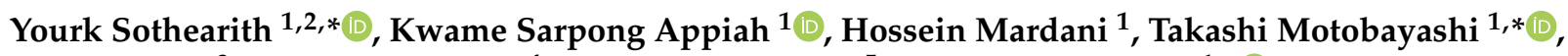 \\ Suzuki Yoko ${ }^{3}$, Khou Eang Hourt ${ }^{4}$, Akifumi Sugiyama ${ }^{5}$ and Yoshiharu Fujii ${ }^{1, *}$ (B) \\ 1 Department of International Environmental and Agricultural Science, Tokyo University of Agriculture and \\ Technology, 3-5-8, Saiwai-cho, Fuchu, Tokyo 183-8509, Japan; ksappiah90@gmail.com (K.S.A.); \\ hmardani26@yahoo.com (H.M.) \\ 2 Department of Biodiversity, Ministry of Environment, Morodok Techcho (Lot 503) Tonle Bassac, \\ Chamkarmorn, Phnom Penh 12301, Cambodia \\ 3 Aromatic Repos, AHOLA, A2 Soleil Jiyugaoka, 1-21-3, Jiyugaoka, Meguro, Tokyo 152-0035, Japan; \\ yoko86252539@gmail.com \\ 4 National Authority for Preah Vihear, Thomacheat Samdech Techo Hun Sen Village, Sraem Commune, Choam \\ Khsant District, Cheom Ksan 13407, Preah Vihear, Cambodia; khou_eanghourt@yahoo.com \\ 5 Research Institute for Sustainable Humanosphere (RISH), Kyoto University, Uji, Kyoto 611-0011, Japan; \\ akifumi_sugiyama@rish.kyoto-u.ac.jp \\ * Correspondence: thearith.yourk@gmail.com (Y.S.); takarice@cc.tuat.ac.jp (T.M.); yfujii@cc.tuat.ac.jp (Y.F.)
}

check for updates

Citation: Sothearith, Y.; Appiah, K.S.; Mardani, H.; Motobayashi, T.; Yoko, S.; Eang Hourt, K.; Sugiyama, A.; Fujii, Y. Determination of the Allelopathic Potential of Cambodia's Medicinal Plants Using the Dish Pack Method. Sustainability 2021, 13, 9062. https://doi.org/10.3390/su13169062

Academic Editors: Alessandra Durazzo and Marc A. Rossen

Received: 19 June 2021

Accepted: 8 August 2021

Published: 13 August 2021

Publisher's Note: MDPI stays neutral with regard to jurisdictional claims in published maps and institutional affiliations.

Copyright: (c) 2021 by the authors. Licensee MDPI, Basel, Switzerland. This article is an open access article distributed under the terms and conditions of the Creative Commons Attribution (CC BY) license (https:// creativecommons.org/licenses/by/ $4.0 /)$.

\begin{abstract}
Plants produce several chemically diverse bioactive substances that may influence the growth and development of other organisms when released into the environment in a phenomenon called allelopathy. Several of these allelopathic species also have reported medicinal properties. In this study, the potential allelopathic effects of more than a hundred medicinal plants from Cambodia were tested using the dish pack method. The dish pack bioassay method specifically targets volatile allelochemicals. Twenty-five species were found to have significant inhibitory effects on lettuce radicle growth. Eleven different plant families, including Iridaceae (2), Apocynaceae (2), Poaceae (2), Sapindaceae, Araceae, Combretaceae, Orchidaceae, Clusiaceae, Zingiberaceae, Rutaceae and Asparagaceae had the plant species with high inhibitory effects. Allophyllus serrulatus had the highest growth inhibitory effect on lettuce radicles more than $60 \%$, followed by Alocasia macrorrhiza, Iris pallida, Terminalia triptera, Wrightia tomentosa, Cymbidium aloifolium, Garcinia villersiana and Kaempferia parviflora. The candidate species were subjected to further studies to identify the volatile allelochemicals in the volatile constituents.
\end{abstract}

Keywords: allelopathy; allelochemicals; volatile compounds; dish pack method

\section{Introduction}

Biodiversity refers to the variety and variability of life on Earth and plays a vital role in ecological functions. The integrative use of plant biodiversity is one approach to improve food security and sustainable agriculture. Species combinations, such as multi-cropping, inter-cropping, alley farming, rotations, and cover cropping, also have positive effects on crop productivity and yield stability [1]. Interaction among plant species may include the production and release of bioactive substances that directly or indirectly influence the growth and development of other organisms in a phenomenon known as allelopathy [2] The definition was later revised to mean any process involving the secondary metabolites produced by plants, microorganisms, viruses and fungi that influence the growth and development of agricultural and biological systems (excluding animals), including positive and negative effects [3]. The secondary metabolites associated with allelopathy released into the environment through volatilization, leaching, root exudation and the decomposition of plant residues in soil are called allelochemicals. These allelochemicals are found in different parts of various plants, such as leaf, root, rhizome, stem, flower, pollen, fruit 
and seed [4,5]. Allelopathy may play an important role in the biological invasion process in natural ecology. Some plants are not dominant competitors in their natural habitat, yet show strong succession when introduced to new areas [6]. Generally, allelopathy is accepted as a significant ecological factor in determining the structure and composition of plant communities [7].

Despite recent advances in the development of agrochemicals for pest control in modern agriculture, crop yields experience average losses of 35\% worldwide. This is mainly due to pests, pathogens and weeds [8]. Weeds are particularly destructive: approximately 30 to $50 \%$ of producing crops are destroyed if weeds are not controlled in Asia and other continents [9-11]. More than 240 weeds have been found to have allelopathic effects on surrounding plants, whether on the same species (autotoxicity) or on other crops and weed species [12]. However, scientists in many different habitats around the world have demonstrated agrochemical pest control. Numerous allelopathic effects from plant species have been reported. For example, 84 out of 245 plant species in the Sino-Japan floristic region have been shown to cause significant inhibitory activity; of these, 10 species showed the strongest effects [13]. The evaluation of the allelopathic potential of 83 Iranian medicinal plants found more than $80 \%$ root growth suppression of lettuce by Peganum harmala, Berberis vulgaris, Artemisia aucheri and Ferulago angulate [14]. The evaluation of allelopathic potential in medicinal plant species used in Ghana found that 75 out of 183 medicinal plant species caused a significant inhibition of lettuce radicle growth through leaf leachates [15]. Identified and isolated bioactive compounds (allelochemicals) from plants are therefore important sources for alternative sustainable and eco-friendly weed control strategies [16], especially given that organic products have increased in popularity over the last decade [17]. The secondary metabolites present in medicinal plants are thought to have relatively strong allelopathic activity. Moreover, analyzing medicinal plants to find new natural compounds is easier than analyzing other plants [2,18-20]. Some bioactive substances, including ferulic, coumaric, vanillic, caffeic and chlorogenic acids in medicinal plants have been found to inhibit plant growth [21,22]. By using the sandwich method, the previous study identified more than fifty medicinal plants with allelopathic potentials through leachates [23]. This study, therefore, collected different parts of some medicinal plants from northwestern Cambodia to examine allelopathic effects using the dish pack method under laboratory conditions.

\section{Materials and Methods}

\subsection{Material}

All the collected medicinal plant samples were oven-dried at $60{ }^{\circ} \mathrm{C}$ for $3 \mathrm{~h}$ at target areas and transferred to the Laboratory of the Department of International Environment and Agriculture, Tokyo University of Agriculture and Technology, Japan to test their allelopathic activities. Lettuce (Lactuca sativa L.) was used as a test plant material in bioassay due to its reliability in germination and its susceptibility to inhibitory and stimulatory chemicals [24].

\subsection{Dish Pack Method}

The dish pack method was adopted for the analysis of volatile allelochemicals of plant species. Most importantly, it allowed us to obtain very quick results, as shown in Figure 1 [25]. Therefore, it was applied to screen all collected medicinal plant species with possibly volatile substances that could influence (promote or inhibit) the growth of lettuce. Multi-well plastic dishes with six wells (36 mm $\times 18 \mathrm{~mm}$ each) were used in this experiment. The distances from the centre of the source well (where the plant sample was placed) to the centre of other wells were 41, 58, 82 and $92 \mathrm{~mm}$. The source well was filled with $200 \mathrm{mg}$ of oven-dried plant materials. Filter papers were laid in the other wells, then $0.75 \mathrm{~mL}$ of distilled water was added to each well that contained filter paper. The control treatment did not contain any plant sample in the source well. Seven lettuce seeds were placed on the filter paper in each well. The multi-well dishes were tightly sealed using cellophane tape to avoid desiccation and the loss of volatile compounds. To exclude 
light, aluminium foil was wrapped around the dishes and placed in an incubator (NTS Model MI-25S) at $25^{\circ} \mathrm{C}$ for three days. With three replications, the radicle and hypocotyl lengths of lettuces were measured and recorded; they were then compared to the lettuce in the control during analysis. The degree of inhibition was estimated by the relationship between lettuce seedling growth inhibition and its distance from the source well.

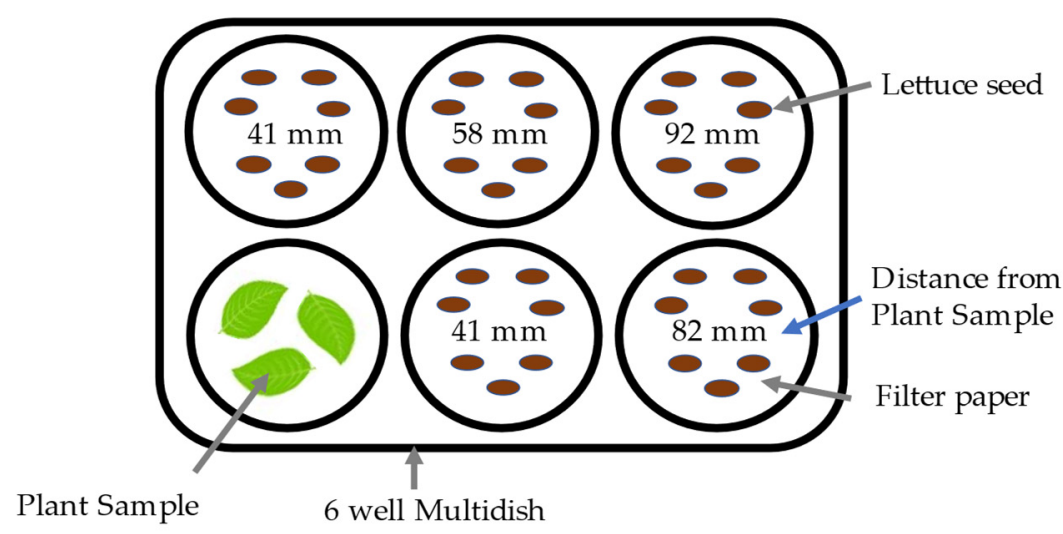

Figure 1. Dish pack method, multi-well plastic plate filled with plant sample and lettuce seeds in each well to test plant allelopathy through volatile substances.

\subsection{Statistic Analysis}

The treatment tested was arranged in a complete randomized design with three replicates. Statistical analysis of the experimental data was conducted with Microsoft Excel 2010. The means, standard deviations (SDs), and SD variances (SDVs) were also evaluated.

$$
\text { Inhibitory }=100-\frac{(\text { Average length of treatment radicle } / \text { hypocotyl })}{(\text { Average length of control radicle/hypocotyl })} \times 100 \text {. }
$$

\section{Results}

The inhibition effects on the radicle and hypocotyl of lettuce seedlings from 195 medicinal plants using the dish pack bioassay method are shown in Table 1. The allelopathic effects of the collected medicinal plants were presented either as the promotion or inhibition of lettuce growth on the radicle and hypocotyl, which ranged from $-19.2 \%$ to $68.6 \%$ and $-30.2 \%$ to $67.3 \%$, respectively. The negative value for the lettuce radicle growth indicates the stimulatory effects compared to the control. The study found several strong candidate species: 25 species from different plant families showed a significant inhibition of lettuce radicle growth among the tested plants. These species came from 11 different families, including Iridaceae (two), Apocynaceae (two), Poaceae (two), Sapindaceae, Araceae, Combretaceae, Orchidaceae, Clusiaceae, Zingiberaceae, Rutaceae and Asparagaceae. However, only Allophyllus serrulatus inhibited more than $60 \%$ on lettuce radicle growth among the tested plants. Radicle growth inhibition in the range of 20-30\% occurred in seven species: Alocasia macrorrhiza, Iris pallida, Terminalia triptera, Wrightia tomentosa, Garcinia villersiana, Cymbidium aloifolium and Kaempferia parviflora. Ten further species, Harrisonia perforate, Eleutherine bulbosa, Imperata cylindrica, Peliosanthes teta, Willughbeia edulis, Eleusine indica, Spatholobus parviflorous, Asplenium nidus, Drynaria quercifolia and Croton oblongifolius demonstrated lettuce radicle inhibitory effects of between 15 and $20 \%$. The lowest effects on lettuce radicle growth were Kaempferia galanga, Afzelia xylocarpa, Zingiber purpureum, Careya sphaerica, Congea tomentosa, Pseuderanthemum latifolium and Ventilago cristata. 
Table 1. The radicle and hypocotyl inhibition percentages of lettuce seedlings grown using the dish pack method.

\begin{tabular}{|c|c|c|c|c|c|c|c|}
\hline \multirow{3}{*}{ Scientific Name } & \multirow{3}{*}{ Plant Families } & \multirow{3}{*}{ Part Used } & \multicolumn{4}{|c|}{ Inhibition Activity (\%) } & \multirow{3}{*}{ Criteria } \\
\hline & & & \multicolumn{2}{|c|}{ Average at $41 \mathrm{~mm}$} & \multicolumn{2}{|c|}{ Average for Whole Wells } & \\
\hline & & & $\mathbf{R}$ & $\mathbf{H}$ & $\mathbf{R}$ & $\mathbf{H}$ & \\
\hline Allophyllus serrulatus Radlk & Sapindaceae & Leaf & 68.6 & 67.3 & 63.1 & 63.1 & $* * *$ \\
\hline Alocasia macrorrhiza (L.) G.Don & Araceae & Tuber & 23.4 & -5.22 & 20.5 & -5.62 & $* * *$ \\
\hline Iris pallida Lam. & Iridaceae & Rhizome & 22.1 & 6.71 & 14.3 & 7.34 & $* * *$ \\
\hline Terminalia triptera Stap f & Combretaceae & Stem & 21.3 & 9.22 & 13.7 & -1.82 & $* *$ \\
\hline Wrightia tomentosa Roem-Schult & Apocynaceae & Stem & 21.1 & 9 & 15.9 & 2.12 & $* *$ \\
\hline Garcinia villersiana Pierre & Clusiaceae & Stem & 20.4 & 1.32 & 16.2 & 2.11 & $* *$ \\
\hline Cymbidium aloifolium (Linn) Swartz. & Orchidaceae & Leaf & 20.4 & 14.2 & 10.7 & 12.4 & $* *$ \\
\hline Kaempferia parviflora Wall. ex Baker & Zingiberaceae & Rhizome & 20.2 & 8.11 & 8.72 & 4.12 & $* *$ \\
\hline Harrisonia perforata Merr. & Rutaceae & Bark & 19.8 & -4.72 & -0.92 & -4.53 & $* *$ \\
\hline Eleutherine bulbosa (Mill.) Urb. & Iridaceae & Flower & 18.7 & 2.42 & 16.7 & 5.62 & ** \\
\hline Imperata cylindrica Beauv & Poaceae & Leaf & 18.2 & 15.2 & 12.4 & 9.91 & $* *$ \\
\hline Peliosanthes teta Andrew & Asparagaceae & Leaf & 17.4 & 13.4 & -0.54 & 6.32 & $* *$ \\
\hline Willughbeia edulis Roxb. & Apocynaceae & Stem & 17.2 & 9.2 & 8.92 & 1.83 & $* *$ \\
\hline Eleusine indica (L) Gaertn & Poaceae & Leaf & 17 & 1.34 & 13.9 & 7.32 & $* *$ \\
\hline Spatholobus parviflorous Kuntz. & Fabaceae & Stem & 16.4 & 6.92 & 10.9 & 0.81 & * \\
\hline Asplenium nidus L. & Aspleniaceae & Leaf & 15.7 & 4.77 & 9.14 & 7.42 & * \\
\hline Drynaria quercifolia (L.) J Sm & Polypodiaceae & Leaf & 15.3 & -5.92 & 9.12 & -5.91 & * \\
\hline Croton oblongifolius Roxb. & Euphorbiaceae & Leaf & 15.2 & 7.6 & 9.32 & 6.63 & $*$ \\
\hline Kaempferia galanga Linn. & Zingiberaceae & Rhizome & 14.8 & 17.4 & 8.41 & 11.2 & * \\
\hline Afzelia xylocarpa (Kurz) Craib. & Fabaceae & Bark & 14.6 & -8.9 & 7.72 & -5.31 & * \\
\hline Zingiber purpureum Roscoe. & Zingiberaceae & Rhizome & 13.9 & 19.5 & 4.62 & 11.6 & * \\
\hline Careya sphaerica Roxb. & Lecythidaceae & Bark & 12.7 & -3.12 & 13.4 & 3.12 & * \\
\hline Congea tomentosa Roxb. & Lamiaceae & Stem & 12.4 & 21.8 & 0 & 12.1 & $*$ \\
\hline Pseuderanthemum latifolium (Vahl) B. Hansen & Acanthaceae & Leaf & 12.3 & 1.4 & 2.33 & -3.74 & * \\
\hline Ventilago cristata Pierre & Rhamnaceae & Stem & 12.3 & 0 & 17.6 & 1.82 & * \\
\hline Sterculia foetida Linn & Sterculiaceae & Stem & 11.5 & -28.4 & 5.07 & -19.1 & \\
\hline Croton lachnocarpus Benth. & Euphorbiaceae & Leaf & 11.4 & -5.21 & 19.1 & 4.31 & \\
\hline Zingiber ottensii Valeton & Zingiberaceae & Rhizome & 11.4 & 57.1 & -0.92 & 41.6 & \\
\hline Ervatamia microphylla Kerr. & Apocynaceae & Leaf & 11.3 & 8.8 & 5.61 & 1.82 & \\
\hline Vitex pubescens Vahl. & Lamiaceae & Stem & 11.1 & 12.2 & 5.12 & 8.93 & \\
\hline Morinda tomentosa Roth & Rubiaceae & Stem & 11 & 11.5 & 6.2 & 10.9 & \\
\hline Hoya diversifolia Blume & Asclepiadaceae & Leaf & 10.1 & 2.7 & 9.12 & 4.71 & \\
\hline Uvaria rufa Blume & Annonaceae & Stem & 9.8 & 15.1 & -1.93 & 7.74 & \\
\hline Scoparia dulcis L & Plantaginaceae & Stem & 9.42 & 6.94 & 4.12 & 0 & \\
\hline Polyalthia evecta (Pierre) Finet et Gagnep. & Annonaceae & Stem & 9.23 & -4.43 & 7.04 & -2.21 & \\
\hline Litchi chinensis Sonn & Sapindaceae & Bark & 9.04 & -7.71 & 2.82 & -5.91 & \\
\hline Artocarpus rigidus Blume & Moraceae & Bark & 8.71 & 6.72 & 5.22 & 0.93 & \\
\hline Kalanchoe Integra Kuntze. & Crassulaceae & Stem & 8.7 & -0.94 & 9 & 2.24 & \\
\hline Hymenocardia punctata Wall. ex Lindl. & Euphorbiaceae & Stem & 8.42 & 1.15 & 3.07 & 4.12 & \\
\hline Zizyphus cambodiana Pierre & Rhamnaceae & Stem & 8.33 & 3.63 & 4.62 & -2.21 & \\
\hline Coptosapelta flavescens Korth. & Rubiaceae & Stem & 8.21 & 2.72 & 6.74 & -2.91 & \\
\hline Ochna integerrima (Lour) Merr. & Ochnaceae & Stem & 8.14 & 7.31 & -1.43 & 2.72 & \\
\hline Curcuma aromatica Salisb. & Zingiberaceae & Leaf & 8.04 & 2.33 & 2.91 & -0.82 & \\
\hline Sindora siamensis Teysm. & Fabaceae & Bark & 8.02 & -0.22 & 0.52 & 3.51 & \\
\hline Suregada multiflora Baill. & Euphorbiaceae & Stem & 8 & -1.64 & 9.61 & -1.83 & \\
\hline Diospyros venosa Wall. & Ebenaceae & Stem & 7.26 & 14.1 & 2.12 & 3.91 & \\
\hline Gnetum montanum Markgr. & Gnetaceae & Stem & 7.23 & -2.6 & -4.04 & -3.3 & \\
\hline Knema globularia Warb. & Myristicaceae & Stem & 7.14 & -7.12 & 5.32 & -4.5 & \\
\hline Stephania rotunda Linn. & Menispermaceae & Tuber & 7.13 & 7.16 & 3.21 & 10.2 & \\
\hline Microcos paniculata L. & Malvaceae & Stem & 7.1 & 6.93 & 3.32 & 6.71 & \\
\hline Costus speciosus (Koenig) J.E.Smith. & Costaceae & Root & 7 & 4.74 & 7 & 4.12 & \\
\hline Amomum xanthioides Wall. & Zingiberaceae & Stem & 6.7 & 8.4 & 6.21 & 0 & \\
\hline Oroxylum indicum (Linn.) Kurz & Bignoniaceae & Bark & 6.62 & 11.6 & 4.43 & 4.61 & \\
\hline Psydrax pergracilis (Bourd.) Ridsdale & Rubiaceae & Stem & 6.32 & 1.8 & 3.13 & -1.82 & \\
\hline Bombax ceiba L. & Malvaceae & Bark & 6.21 & 7.75 & 20.1 & 12.9 & \\
\hline Donax grandis Ridley & Poaceae & Stem & 5.94 & 11.2 & -0.21 & 6.81 & \\
\hline Carallia brachiata (Lour.) Merr & Rhizophoraceae & Bark & 5.86 & 4.54 & 8.54 & 11.1 & \\
\hline Pouzolzia zeylanica (L) Benn. & Urticaceae & Stem & 5.82 & -9.42 & 4.61 & -6.32 & \\
\hline Gomphrena celosioides Mart. & Amaranthaceae & Flower & 5.62 & -3.61 & -3.71 & -11 & \\
\hline Crinum latifolium $\mathrm{L}$. & Amaryllidaceae & Bulb & 5.48 & 8.61 & 0.52 & 2.73 & \\
\hline Strychnos wallichiana Steud. Ex DC. & Loganiaceae & Stem & 5.44 & 7.32 & -1.21 & 3.52 & \\
\hline Melastoma villosum $\mathrm{L}$. & Melastomataceae & Stem & 5.33 & -8.73 & 7.21 & -7.64 & \\
\hline Ixora chinensis Lam. & Rubiaceae & Leaf & 5.17 & -1.14 & 3.12 & 2.21 & \\
\hline Eupatorium odoratum (L.) R.M.King \& H.Rob. & Asteraceae & Leaf & 5.1 & 20 & 4.72 & 12.2 & \\
\hline
\end{tabular}


Table 1. Cont

\begin{tabular}{|c|c|c|c|c|c|c|c|}
\hline \multirow{3}{*}{ Scientific Name } & \multirow{3}{*}{ Plant Families } & \multirow{3}{*}{ Part Used } & \multicolumn{4}{|c|}{ Inhibition Activity (\%) } & \multirow{3}{*}{ Criteria } \\
\hline & & & \multicolumn{2}{|c|}{ Average at $41 \mathrm{~mm}$} & \multicolumn{2}{|c|}{ Average for Whole Wells } & \\
\hline & & & $\mathbf{R}$ & $\mathbf{H}$ & $\mathbf{R}$ & $\mathbf{H}$ & \\
\hline Dipterocarpus tuberculatus Roxb & Dipterocarpaceae & Stem & 4.88 & -5.72 & 3.91 & -1.82 & \\
\hline Licuala spinosa Wurmb & Arecaceae & Root & 4.84 & -30.2 & 0.23 & -15.2 & \\
\hline Nepenthes kampotiana Lecomte & Nepenthaceae & Flower & 4.83 & -10.6 & 3.12 & -11.1 & \\
\hline Smilax ovalifolia Roxb. & Smilacaceae & Stem & 4.8 & 14.8 & -0.42 & 3.64 & \\
\hline Cnestis palala (Lour.) Merr. & Connaraceae & Leaf & 4.67 & 7.72 & -4.51 & -0.92 & \\
\hline Smilax china L. & Smilacaceae & Stem & 4.65 & 13.1 & 1.62 & 11.2 & \\
\hline Dillenia pentagyna Roxb & Dilleniaceae & Stem & 4.58 & -12.7 & 1.21 & -7.62 & \\
\hline Gonocaryum lobianum (Miers) Kurz & Icacinaceae & Stem & 4.55 & -13.8 & 2.23 & -6.74 & \\
\hline Physalis angulata L. & Solanaceae & Root & 4.52 & 0.82 & 2.71 & 7.42 & \\
\hline Irvingia malayana Olive. Ex Benn. & Irvingiaceae & Bark & 4.42 & -4.62 & 6.12 & 0.4 & \\
\hline Dioscorea hispida Dennst. & Dioscoreaceae & Tuber & 4.16 & 8.44 & 0 & 7.82 & \\
\hline Lagerstroemia calyculata Kurz. & Lythraceae & Bark & 4 & 3.81 & 7.24 & 6.34 & \\
\hline Syzygium polyanthum (Wight) Walp. & Myrtaceae & Bark & 3.9 & 5.64 & -4.93 & -0.91 & \\
\hline Streblus asper Lour. & Moraceae & Stem & 3.81 & -6.72 & 4 & -2.23 & \\
\hline Tinospora crispa (Linn) Miers ex Hook. & Menispermaceae & Stem & 3.77 & -3.13 & -30.9 & 0 & \\
\hline Anthocephalus chinensis (Lam.) & Rubiaceae & Bark & 3.46 & -1.53 & 7.61 & -0.72 & \\
\hline Borassus flabellifera Linn & Arecaceae & Root & 3.42 & -7.31 & 7.21 & -2.81 & \\
\hline Cassia alata L. & Fabaceae & Stem & 3.28 & -9.2 & -6.32 & -0.91 & \\
\hline Mangifera duperreana Pierre & Anacardiaceae & Bark & 3.23 & -2.12 & 11.6 & 10.3 & \\
\hline Tetracera scendens (L.) Merr. & Dilleniaceae & Leaf & 2.83 & 4.34 & 3.62 & 5.91 & \\
\hline Lygodium flexuosum (L.) SW. & Lygodiaceae & Leaf & 2.62 & -4.52 & 2 & -4.12 & \\
\hline Blumea balsamifera DC. & Asteraceae & Leaf & 2.41 & 19.6 & 7.52 & 14.3 & \\
\hline Diospyros decandra Lour & Ebenaceae & Bark & 2.37 & -4.1 & 10.3 & 3.91 & \\
\hline Bauhinia bassacensis Pierre & Fabaceae & Stem & 2.12 & 10.7 & 9.73 & 6.42 & \\
\hline Clerodendrum schmidtii C.B.Clarke & Lamiaceae & Stem & 1.97 & 7.74 & -4.11 & -11.3 & \\
\hline Elaeocarpus stipularis Blume & Elaeocarpaceae & Stem & 1.92 & -16.7 & 4.62 & -11.4 & \\
\hline Memecylon laevigalum Blume & Melastomataceae & Stem & 1.72 & 3.42 & 0.41 & 6.43 & \\
\hline Illigera rhodantha Hance. & Hernandiaceae & Stem & 1.58 & -7.62 & -2 & -6.73 & \\
\hline Phyllanthus amarus Schum.ct Thonn. & Phyllanthaceae & Stem & 1.53 & 1.12 & 12.2 & 4.91 & \\
\hline Ficus hispida $\mathrm{L}$. & Moraceae & Stem & 1.26 & 8.81 & -0.21 & 9 & \\
\hline Ancistrocladus tectorius (Lour.) Merr. & Ancistrocladaceae & Stem & 1.18 & 15.1 & -0.54 & 10.2 & \\
\hline Moringa oleifera Lamk & Moringaceae & Bark & 1.16 & -2.92 & -1.21 & -0.83 & \\
\hline Melodorum fruticosum Lour & Annonaceae & Stem & 1.15 & -1.65 & -0.32 & 2.7 & \\
\hline Peltophorum dasyrhachis (Miq.) Kurz & Fabaceae & Bark & 1.12 & 11.7 & -9.12 & 7.31 & \\
\hline Prismatomeris tetrandra (Roxb.) K.Schum & Rubiaceae & Stem & 1.1 & -4.91 & 7.9 & -8.12 & \\
\hline Dipterocarpus obtusifolius Teijsm.-ex-Miq & Dipterocarpaceae & Stem & 0.82 & 4.52 & -3.21 & -0.93 & \\
\hline Macaranga triloba (Blume) Muell.Arg. & Euphorbiaceae & Stem & 0.76 & 0.23 & 4.12 & -1.81 & \\
\hline Typhonium trilobatum Schott & Araceae & Stem & 0.65 & -2.83 & -4.31 & -6.42 & \\
\hline Scindapsus officinalis (Roxb.) Schott & Araceae & Stem & 0.54 & 4.81 & -2.12 & 1.31 & \\
\hline Erythroxylum cambodianum Pierre & Erythroxylaceae & Stem & 0.54 & -2.54 & -1.63 & 0.52 & \\
\hline Spirolobium cambodianum Baill. & Apocynaceae & Stem & 0.52 & -1.22 & 1 & -5.24 & \\
\hline Caesalpinia sappan Linn. & Fabaceae & Bark & 0.37 & 16.3 & -0.52 & 9.73 & \\
\hline Melastoma mormale (Kuntze) Merr. & Melastomataceae & Stem & 0.33 & 2.32 & 1 & 11.9 & \\
\hline Heliotropium indicum $\mathrm{L}$. & Boraginaceae & Leaf & 0.22 & -9.44 & -2.81 & -8.82 & \\
\hline Eurycoma longifolia Jack & Simaroubaceae & Bark & 0.17 & 4.51 & -2.72 & 3.31 & \\
\hline Shorea roxburgii G Don & Dipterocarpaceae & Bark & 0.15 & 3.21 & -0.71 & 1.82 & \\
\hline Plumbago zeylanica L. & Plumbaginaceae & Stem & 0 & 2.84 & -2.24 & 3.22 & \\
\hline Rauwenhoffia siamensis Scheff & Annonaceae & Stem & -0.1 & -9.42 & -7.2 & -8.21 & \\
\hline Scheffera elliptaca (Blume) Harms. & Araliaceae & Stem & -0.22 & 5.35 & 1.61 & 4.52 & \\
\hline Manilkara hexandra (Roxb.) Dubard & Sapotaceae & Leaf & -0.25 & 0.2 & -1.72 & 2.1 & \\
\hline Senna siamea Lam & Fabaceae & Leaf & -0.27 & -18.1 & -2.71 & -14.2 & \\
\hline Fagraea fragrans Roxb. & Loganiaceae & Stem & -0.63 & 14.4 & 3.31 & 10.5 & \\
\hline Dracaena loureiri (Gagnep.) & Asparagaceae & Bark & -0.65 & 12.5 & 1.21 & 11.1 & \\
\hline Couroupita guianensis Aubert & Lecythidaceae & Flower & -1.42 & -7.12 & -3.9 & 1.4 & \\
\hline Cleistanthus tomentosus Hance & Euphorbiaceae & Stem & -1.45 & -8.05 & 0.92 & -4.12 & \\
\hline Albizia lebbek (L.) Benth. & Mimosaceae & Stem & -1.57 & 13.4 & -5.81 & 13.6 & \\
\hline Fhyllanthus emblica L. & Euphorbiaceae & Stem & -1.63 & -9.12 & -0.52 & -8.53 & \\
\hline Alpinia conchigera Grulf & Zingiberaceae & Leaf & -1.72 & -0.2 & 4.14 & 0.91 & \\
\hline Ficus sagitta Vahl. & Moraceae & Leaf & -1.94 & -15.5 & -0.52 & -6.31 & \\
\hline Derris scandens (Roxb.) Benth. & Fabaceae & Stem & -2 & -4.13 & 8.62 & -0.91 & \\
\hline Andrographis paniculata (Burm.f.) & Acanthaceae & Leaf & -2.14 & 1.1 & 9.9 & -1.31 & \\
\hline Pandanus capusii Marc & Pandanaceae & Root & -2.33 & -13.4 & -8 & -15.3 & \\
\hline Streptocaulon juventas Merr. & Apocynaceae & Stem & -2.37 & 3.31 & 0.22 & 3.83 & \\
\hline Dioscorea bulbifera L. & Discoreaceae & Tuber & -2.52 & 0.44 & -1.41 & -2.52 & \\
\hline
\end{tabular}


Table 1. Cont

\begin{tabular}{|c|c|c|c|c|c|c|c|}
\hline \multirow{3}{*}{ Scientific Name } & \multirow{3}{*}{ Plant Families } & \multirow{3}{*}{ Part Used } & \multicolumn{4}{|c|}{ Inhibition Activity (\%) } & \multirow{3}{*}{ Criteria } \\
\hline & & & \multicolumn{2}{|c|}{ Average at $41 \mathrm{~mm}$} & \multicolumn{2}{|c|}{ Average for Whole Wells } & \\
\hline & & & $\mathbf{R}$ & $\mathbf{H}$ & $\mathbf{R}$ & $\mathbf{H}$ & \\
\hline Gnetum latifolium Blume & Gnetaceae & Stem & -2.55 & 4.73 & -7.41 & -0.44 & \\
\hline Entada phaseoloides Merr. & Fabaceae & Fruit & -2.6 & 3.24 & -4.51 & -7.23 & \\
\hline Mallotus paniculatus (Lam.) Mull.Arg. & Euphorbiaceae & Stem & -2.64 & -4.6 & -7.92 & -2.92 & \\
\hline Schleicheria oleosa (Lour.) Oken. & Sapindaceae & Stem & -2.8 & 0.5 & -11.2 & -1.91 & \\
\hline Elephantopus scaber L. & Asteraceae & Leaf & -3 & -13.3 & -4.12 & -11.6 & \\
\hline Solanum toroum Swartz & Solanaceae & Stem & -3.11 & 0 & 5.31 & 2.11 & \\
\hline Glycosmis pentaphylla (Retz) Correa & Rutaceae & Stem & -3.24 & -7.32 & -2.53 & -3.81 & \\
\hline Acalypha boehmerioides Miq. & Euphorbiaceae & Leaf & -3.41 & -10.2 & -6.12 & -11.4 & \\
\hline Lagerstroemia floribunda Jack. & Lythraceae & Bark & -3.57 & -5.4 & -4.31 & -4.9 & \\
\hline Micromelum falcatum (Lour.) Tanak & Rutaceae & Stem & -3.58 & -10.4 & -6.33 & -10.4 & \\
\hline Ficus benjamina L. & Moraceae & Stem & -4.1 & -3 & 3.7 & 6.12 & \\
\hline Hydnophytum formicarium Jack & Rubiaceae & Tuber & -4.12 & 9.53 & -6.04 & 6.6 & \\
\hline Capparis micracantha DC. & Capparaceae & Stem & -4.21 & 8.34 & 0.23 & 6.92 & \\
\hline Terminalia corticosa Pierre. & Combretaceae & Bark & -4.24 & 2.83 & -11.8 & -3.72 & \\
\hline Pteridium aquilinum (L) Kuhm. & Dennstaedtiaceae & Leaf & -4.45 & 2.62 & -5.61 & 5.32 & \\
\hline Sida rhombifolia L. & Malvaceae & Root & -4.48 & -12.9 & -5.4 & -7.81 & \\
\hline Cananga latifolia Finet et Gagnep & Annonaceae & Stem & -4.51 & 1.2 & -1 & 3.31 & \\
\hline Parinari anamensis Hance & Chrysobalanaceae & Bark & -4.56 & -1.64 & 0.2 & -0.92 & \\
\hline Gardenia philastrei Pierre-ex-Pit. & Rubiaceae & Stem & -4.72 & -4.21 & -6.91 & -13.4 & \\
\hline Parameria laevigata (Juss.) Moldenke & Apocynaceae & Bark & -4.74 & -0.72 & -7.52 & -3.21 & \\
\hline Alstonia scholaris $\mathrm{R}-\mathrm{Br}$ & Apocynaceae & Bark & -5.1 & 3.37 & -5.91 & 0.9 & \\
\hline Tiliacora triandra Diels & Menispermaceae & Stem & -5.15 & -3.83 & -3.81 & -3.34 & \\
\hline Dracaena angustifolia Roxb. & Asparagaceae & Leaf & -5.3 & 2.65 & -6.74 & -1.06 & \\
\hline Holarrhena curtisii King \&Gamble & Apocynaceae & Leaf & -5.35 & -1.44 & -7.23 & 0 & \\
\hline Parabarium micranthum (A.DC.) Pierre & Apocynaceae & Leaf & -5.41 & -4.82 & -1.72 & -6.51 & \\
\hline Dialium cochinchinense Pierre & Fabaceae & Bark & -5.71 & 13.3 & -7.72 & 5.43 & \\
\hline Jasminum nobile C.B.Clarke & Oleaceae & Stem & -5.78 & -14.3 & -2.91 & -2.7 & \\
\hline Melaleuca cajuputi Powell & Myrtaceae & Leaf & -5.79 & -4.84 & 1.31 & 0.32 & \\
\hline Hymenodictyon excelsum (Roxb) w. & Rubiaceae & Leaf & -5.8 & -7.11 & 3.11 & -2.81 & \\
\hline Derris elliptica (Wall.) Benth. & Fabaceae & Stem & -6.21 & 7.72 & -1.62 & 5.9 & \\
\hline Leea rubra $\mathrm{Bl}$ & Vitaceae & Stem & -6.23 & -8.32 & -2.11 & -13.8 & \\
\hline Rhodomyrtus tomentosa (Ait) Hassk & Myrtaceae & Leaf & -6.28 & 14.2 & -6.08 & 6.31 & \\
\hline Brucea javanica (Linn) Merr. & Simaroubaceae & Stem & -6.31 & -8.33 & -4.81 & -10.1 & \\
\hline Mimosa pudica Linn. & Fabaceae & Leaf & -6.6 & -5.64 & 0.72 & -0.7 & \\
\hline Lygodium conforme C. Chr. & Lygodiaceae & Leaf & -7.4 & 5.42 & 1.41 & 8.6 & \\
\hline Adina cordifolia Hok. F & Rubiaceae & Stem & -7.5 & 2.81 & -10.7 & 0 & \\
\hline Aquilaria crassna Pierr. & Thymeleaceae & Root & -7.52 & -5.62 & -0.92 & -2.71 & \\
\hline Ficus pumila L. & Moraceae & Leaf & -7.55 & 12.2 & -5.72 & 7.71 & \\
\hline Scleria terrestris (L.) Fassett & Cyperaceae & Leaf & -7.72 & -25.6 & -2.61 & -15.7 & \\
\hline Calamus rudentum Lour. & Arecaceae & Stem & -7.82 & -12.1 & -0.8 & -4.04 & \\
\hline Neonauclea sessilifolia (Roxb.)Merr. & Rubiaceae & Bark & -7.9 & 8.93 & -4.3 & -2.51 & \\
\hline Broussonetia papyrifera (L.) L'Hér. ex Vent. & Urticaceae & Stem & -7.9 & -1.9 & -10.1 & -3.2 & \\
\hline Diospyros nitida Merr. & Ebenaceae & Stem & -8 & 0.92 & -3 & 3.81 & + \\
\hline Zizyphus oeniplia Mill & Rhamnaceae & Stem & -8.12 & -8.42 & -9.08 & -5.62 & + \\
\hline Cyclea barbata Miers & Menispermaceae & Leaf & -8.41 & -5.95 & -9.11 & -3.63 & + \\
\hline Dillenia ovata Wall. ex Hook.f. & Dilleniaceae & Bark & -8.44 & -6.14 & -9.8 & -9.43 & + \\
\hline Homonoia riparia Lour. & Euphorbiaceae & Bark & -8.49 & 8.34 & -6.32 & 2.31 & + \\
\hline Colona auriculata (Desv.) Craib & Tiliaceae & Stem & -9.71 & -6.08 & -11.5 & -8.6 & + \\
\hline Mussaenda cambodiana Pirrl ex Pit & Rubiaceae & Stem & -9.77 & -0.62 & -10.6 & -6.83 & + \\
\hline Pandanus tectorius Parkinson ex Du Roi & Pandanaceae & Leaf & -10 & -8.34 & -7.05 & -10.1 & + \\
\hline Cyperus rotundus Linn. & Cyperaceae & Leaf & -10.3 & -19.2 & -6.91 & -15.7 & + \\
\hline Aganosma marginata G. Don & Apocynaceae & Stem & -10.7 & -0.92 & -15.2 & 2.61 & + \\
\hline Mesua ferrea $\mathrm{L}$ & Calophyllaceae & Leaf & -10.7 & 12.5 & -10.2 & 5.92 & + \\
\hline Lindernia crustacea (L.) F.Muell. & Linderniaceae & Stem & -10.9 & 5.63 & -1.44 & 7.82 & + \\
\hline Zanthoxylum rhetsa DC. & Rutaceae & Bark & -11.4 & 9.56 & -11.4 & -7.11 & + \\
\hline Walsura villosa Wall. Ex Hiern. & Meliaceae & Bark & -12.4 & -5.24 & -9.71 & -1.32 & + \\
\hline Acacia harmandiana (Pierre) Gagnep. & Fabaceae & Bark & -12.5 & -13.1 & -15.7 & -4.1 & ++ \\
\hline Ampelocissus matinii Planch & Vitaceae & Stem & -13.1 & 0.52 & -9.91 & -0.92 & ++ \\
\hline Euphorbia hirta Linn. & Euphorbiaceae & Leaf & -13.3 & -20.8 & -13.5 & -15.7 & ++ \\
\hline Madhuca butyrospermoides A.Chev. & Sapotaceae & Bark & -13.3 & -2.1 & -7.94 & -1.44 & ++ \\
\hline
\end{tabular}


Table 1. Cont.

\begin{tabular}{|c|c|c|c|c|c|c|c|}
\hline \multirow{3}{*}{ Scientific Name } & \multirow{3}{*}{ Plant Families } & \multirow{3}{*}{ Part Used } & \multicolumn{4}{|c|}{ Inhibition Activity (\%) } & \multirow{3}{*}{ Criteria } \\
\hline & & & \multicolumn{2}{|c|}{ Average at $41 \mathrm{~mm}$} & \multicolumn{2}{|c|}{ Average for Whole Wells } & \\
\hline & & & $\mathbf{R}$ & $\mathbf{H}$ & $\mathbf{R}$ & $\mathbf{H}$ & \\
\hline Millingtonia hortensis Linn & Bignoniaceae & Stem & -13.4 & -5.42 & -9.71 & 2.23 & ++ \\
\hline Phyllanthus reticulatus Poir & Euphorbiaceae & Stem & -14.1 & -14.1 & -10.5 & -12.9 & ++ \\
\hline Randia tomentosa $\mathrm{Bl}$. & Rubiaceae & Stem & -15.4 & -3.61 & -12.4 & 1.41 & ++ \\
\hline Anacardium occidentale Linn & Anacardiaceae & Bark & -15.9 & -14.3 & -10.1 & -6.74 & ++ \\
\hline Salacia chinensis Linn. & Celastraceae & Stem & -15.9 & 2.54 & -12.8 & 6.72 & ++ \\
\hline Ficus hirta Vahl var roxburghii (Miq). & Moraceae & Stem & -16 & -1.72 & -12.8 & -1.33 & ++ \\
\hline Sterculia lychnophora Hance & Sterculiaceae & Stem & -19.2 & -10.2 & -12.1 & -0.42 & +++ \\
\hline
\end{tabular}

Note: Criteria $(*),(* *)$ and $(* *)$ refer to radicle elongation shorter than the mean value plus $1.0(\mathrm{SD}), 1.5(\mathrm{SD})$ and $2(\mathrm{SD})-$ that is, SDV $=12$, 17 and 22 , respectively. + Criteria $(+),(++)$ and $(+++)$ refer to radicle elongation longer than the mean value minus $1.0(\mathrm{SD}), 1.5(\mathrm{SD})$ and $2(\mathrm{SD})$ - that is, $\mathrm{SDV}=-8,-13$ and -18 , respectively.

\section{Discussion}

In the Sapindaceae plant family, Allophylus serratus, a large shrub found all over India, showed a stronger inhibition activity through volatile compounds than Litchi chinensis and Schleicheria oleosa. Allophylus serratus is used as an anti-inflammatory and carminative due to its strong pharmacological activity. This plant is also used to treat numerous medical conditions, such as elephantiasis, oedema and bone fractures, as well as several gastrointestinal disorders, including dyspepsia, anorexia and diarrhea [26]. Bioactive substances contained in Allophylus serratus include phenolic compounds, flavonoids, tanning substances, steroids, alkaloids and saponins were reported [27]. Other compounds isolated from Allophylus serratus, such as quercetin, pinitol, luteolin-7-O- $\beta$-D-glucopy-ranoside, rutin and apigenin-4-O- $\beta$-D-glucoside. However, only rutin showed an increase in osteoblast mineralization, as assessed by alizarin extraction; its use has been suggested for menopausal osteoporosis [28].

Another interesting species is Alocasia macrorrhiza (common name Elephant Ear Taro), a giant plant with distinctive leaves, which is mostly used for ornamental purposes and belongs to the Araceae family [29]. Elephant Ear Taro is a massive herb formed by a thick erect trunk in large plants and up to $4 \mathrm{~m}$ in height; its leaves are held erect with petioles (leaf stalks) that are up to $130 \mathrm{~cm}$ long [30]. It has antifungal, antidiuretic, laxative, antitubercular and antioxidant properties; it also features other compounds such as flavonoids, oxalic acid, cyanogenic glycosides, alocasin, cholesterol, amino acids, gallic acid, malic acid, ascorbic acid, succinic acid, glucose, fructose, sucrose and beta-lectins [31]. Additionally, 14 compounds have been isolated and identified from giant taro, including 5 new lignan amides, 1 new monoindole alkaloid and 8 known compounds [32].

Iris pallida from the Iridaceae family also showed potential inhibitory effects. Iris contains up to 80 genera and 300 species that are distributed worldwide; it is abundant and diversified in the regions of Southern Africa and Asia. Many of these species are common ornamental plants [33]. Iris pallida, known as the sweet iris, is a perennial herb native to the Dalmatian coast, Croatia; it is mostly cultivated for its essential oils and use in aromatherapy and traditional medicine [34,35]. The rhizomes of Iris pallida found to have strong allelopathic activity contain the isoflavones irigenin, iristectorigenin A, nigericin, nigricanin, irisflorentin, iriskumaonin methyl ether, irilone, iriflogenin and others [23,36-39]. In total, 16 and 26 volatile components were found from the essential oil of rhizomes and leaves, respectively. Dihydro- $\beta$-irone, $\alpha$-irone, trans-2,6- $\gamma$-irone, $\beta$-isometilionone; benzophenone and other dominant terpenes, including 4-isobutylphenone, benzophenone, hexahydrofarnesyl acetone, neofitadien and squalene were also reported [40]. The bioactive substances, including irones in iris rhizomes could offer commercial potential in the form of iris essential oil [41].

In the Rutaceae family, Harrisonia perforata Merr, known as a prickly shrub, is native to China but widely distributed across Southeast Asia. This plant is nearly upright, growing up to $6 \mathrm{~m}$ tall. Several parts of this prickly shrub are gathered from the wild and used locally 
as medicines to treat some diseases, such as dysentery and cholera, and to relieve itching. It is also reported that its root when dried contains antipyretic and anti-inflammatory properties that are used to deal with wound healing and diarrhea [42]. The leaves, fruits, branches and roots of Harrisonia perforate have been reported to contain several chromones, limonoids, triterpenoids and prenylated polyketides, including harrisotone A-E, haperforine A, haperforine E, 12-desacetylhap-erforine A, haperforine $\mathrm{C} 2$, haperforine $\mathrm{F}$, haperforine $\mathrm{G}$, Foritin, harrisonol A, peucenin-7-methyl ether, O-methyla-lloptaeroxylin, perforatic acid, eugenin, saikochromone $\mathrm{A}$, greveichromenol and perforamone $\mathrm{A}-\mathrm{D}$ [43]; $\beta$-sitosterol, obacunone, herteropeucenin-7-methyl ether, perforatic acid and harrisonin [44-47]; and harperforatin, harperfolide and harperamone [48].

\section{Conclusions}

This study presents a preliminary analysis of the potential volatile allelopathic effects of some medicinal plants in Cambodia. The revealed data could help future researchers to isolate and identify volatile allelochemicals to demonstrate bio-herbicides for sustainable weed control. Allophyllus serrulatus, which showed the highest inhibitory effect, was recommended for the further identification and characterization of allelopathic substances.

Author Contributions: Conceptualization, Y.S., K.S.A., H.M. and Y.F.; methodology, K.S.A., H.M. and Y.F.; software, Microsoft Office 2016; validation, T.M., K.S.A. and K.E.H.; Resources, A.S. and Y.F.; Funding acquisition, A.S. and Y.F.; data curation, Y.S. and K.E.H.; writing-initial draft preparation, Y.S.; writing-review and editing, Y.S., K.S.A., S.Y. and Y.F.; supervision, T.M. and Y.F. All authors have read and agreed to the published version of the manuscript.

Funding: This research received funding from the Ministry of Education, Culture, Sports, Science, and Technology (MEXT), Japan. This work was also partly supported by JST CREST Grant Number JPMJCR17O2 and JSPS KAKENHI Grant Number 26304024.

Data Availability Statement: No new data were created or analyzed in this study.

Acknowledgments: The authors thank the Japanese Ministry of Education, Culture, Sports, Science, and Technology (MEXT), JST CREST and JICE/JDS for providing the scholarship to the first author at the Tokyo University of Agriculture and Technology. We also gratefully acknowledge to the Ministry of Environment, the Ministry of Agriculture, Forestry and Fisheries, the Provincial Department of Environment, Siem Reap Province and the Local Community at Phnom Kulen National Park, Cambodia for supporting and assisting in sample collection and transfer for this research study.

Conflicts of Interest: The authors declare no conflict of interest.

\section{References}

1. FAO. Biodiversity for Food and Agriculture Contributing to Food Security and Sustainability in a Changing World. In Proceedings of the Workshop Report, Rome, Italy, 14-16 April 2010.

2. Rice, E.L. Allelopathy, 2nd ed.; Academic Press: New York, NY, USA, 1984.

3. Torres, A.; Oliva, R.M.; Castellano, D.; Cross, P. First World Congress on Allelopathy. In A Science of the Future, SAI; University of Cádiz: Andalusia, Spain, 1996; p. 278.

4. Fujii, Y.; Hiradate, S. Allelopathy: New Concepts and Methodology; Science Publishers Inc.: Enfield, NH, USA, 2007.

5. Turk, M.A.; Tawaha, A.M. Allelopathic effect of black mustard (Brassica nigra L.) on germination and growth of wild oat (Avena fatua L.). Crop Prot. 2003, 22, 673-677. [CrossRef]

6. Driesche, R.G.V.; Bellows, T.S. Pest Origins, Pesticides and the History of Biological Control. In Biological Control; Chapman and Hall: New York, NY, USA, 1996; pp. 1-20.

7. Scrivanti, L.R.; Zunino, M.P.; Zygadlo, J.A. Tagetes minuta and Schinus areira essential oils as allelopathic agents. Biochem. Syst. Ecol. 2003, 31, 563-572. [CrossRef]

8. Oerke, E.C. Centenary Review Crop losses to pests. Agric. Sci. 2006, 144, 31-43. [CrossRef]

9. Swarbrick, B.L.; Mercado, J.T. Weed Science and Weed Control in Southeast Asia. In FAO Plant Production and Protection; Food and Agriculture Organization of the United Nations: Rome, Italy, 1987; p. 81.

10. Oerke, E.C.; Dehne, H.W. Global crop production and the efficacy of crop protection-Current situation and future trends. Eur. J. Plant Pathol. 1997, 103, 203-215. [CrossRef]

11. Karim, S.M.R.; Iqbal, T.M.T.; Islam, N. Relative yields of crops and crop losses due to weed competition in Bangladesh. Pak. J. Sci. Ind. Res. 1999, 41, 318-324. 
12. Colquhoun, J.B. Allelopathy in Weeds and Crops: Myths and Facts. In Proceedings of the Wisconsin Fertilizer, Aglime and Pest Management Conference, Madison, WI, USA, 17-19 January 2006; Volume 45, pp. 318-319.

13. Appiah, K.S.; Zhenhao, L.; Ren, S.Z.; Shiming, L.; Oikawa, Y.; Fujii, Y. Determination of allelopathic potentials in plant species in Sino-Japanese floristic region by sandwich method and dish pack method. Int. J. Basic Appl. Sci. 2015, 4, 381-394. [CrossRef]

14. Mardani, H.; Azizi, M.; Osivand, A.; Fujii, Y. Evaluation of Allelopathic Activity of Iranian Medicinal Plants by Sandwich Method. J. Weed Sci. Technol. 2014, 53, 85.

15. Appiah, K.S. Evaluation of Allelopathic Potentials in Medicinal Plant Species Used in Ghana. Master's Thesis, Tokyo University of Agriculture and Technology, Tokyo, Japan, 2016.

16. Khanh, T.D.; Hong, N.H.; Xuan, T.D.; Chung, I.M. Paddy weed control by medicinal and leguminous plants from Southeast Asia. Crop Prot. 2005, 24, 421-431. [CrossRef]

17. Willer, H.; Kilcher, L. Training Manual for Organic Agriculture: The World of Organic Agriculture. In Statistics and Emerging Trends 2009; IFOM: Bonn, Germany, 2009.

18. Chevallier, A. The Encyclopedia of Medicinal Plants: A Practical Reference Guide to over 550 Key Herbs E Their Medicinal Uses; Dorling Kindersley: London, UK, 1996.

19. Michael, W. Introduction: Biochemistry, Role and Biotechnology of Secondary Metabolites. Annu. Plant Rev. 1999, 2, 1-16.

20. Fujii, Y.; Parvez, S.S.; Parvez, M.M.; Ohmae, Y.; Iida, O. Screening of 239 Medicinal Plant Species for Allelopathic Activity using the Sandwich Method. Weed Biol. Manag. 2003, 3, 233-241. [CrossRef]

21. Modallal, N.M.; Al-Charchafchi, F.M.R. Allelopathic effect of Artemisia harba alba on germination and seedling growth of Anabasis setifera. Pak. J. Biol. Sci. 2006, 9, 1795-1798. [CrossRef]

22. Nazir, T.; Uniyal, A.K.; Todaria, N.P. Allelopathic behavior of three medicinal plant species on traditional agriculture crops of Garhwal Himalaya. India Agrofor. Syst. 2007, 69, 183-187. [CrossRef]

23. Sothearith, Y.; Appiah, K.S.; Motobayashi, T.; Watanabe, I.; Somaly, C.; Sugiyama, A.; Fujii, Y. Evaluation of Allelopathic Potentials from Medicinal Plant Species in Phnom Kulen National Park, Cambodia by the Sandwich Method. Sustainability 2021, 13, 264. [CrossRef]

24. Fujii, Y.; Shibuya, T.; Yasuda, T. Survey of Japanese weed and crops for the detection of water-extractable allelopathic chemicals using Richards' function fitted to lettuce germination test. Weed Res. Jpn. 1990, 35, 362-370.

25. Fujii, Y.; Matsuyama, M.; Hiradate, S.; Shimozawa, H. Dish pack method: A new bioassay for volatile allelopathy. Thymus 2005, 2, 493-497.

26. Dharmani, P.; Mishra, P.K.; Maurya, R.; Chauhan, V.S.; Palit, G. Allophylus serratus: A plant with potential anti-ulcerogenic activity. J. Ethnopharmacol. 2005, 99, 361-366. [CrossRef]

27. Jemal, K.; Sandeep, B.V.; Pola, S.R. Review Article a Review on Medicinal Importance of Allophylus serrattus and Premna tomentosa. Int. J. Curr. Res. 2015, 7, 21034-21039.

28. Kumar, M.; Rawat, P.; Dixit, P.; Mishra, D.; Gautam, A.K. Anti-osteoporotic constituents from Indian medicinal plants. Phytomedicine 2010, 17, 993-999. [CrossRef]

29. Ongpoy, R.C., Jr. The Medicinal Properties of the Alocasia Genus: A Systematic Review. JAASP Res. Pap. $2017,6,1$.

30. Available online: http://www.kew.org/science-conservation/plants-fungi/alocasia-macrorrhizos-elephant-ear-taro (accessed on 10 August 2021).

31. Singh, S.K.; Patel, J.R.; Dangi, A.; Bachle, D.; Katariya, R.K. Review Paper on Alocasia macrorrhiza Indian Medicinal Plant. Eur. J. Pharm. Med. Res. 2017, 4, 366-375.

32. Huang, W.; Li, C.; Wang, Y.; Yi, X.; He, X. Anti-inflammatory lignanamides and monoindoles from Alocasia macrorrhiza. Fitoterapia 2017, 117, 126-132. [CrossRef]

33. Goldblatt, P.; Manning, J.C.; Demissew, S.S. Two new species of Zygotritonia Mildbr. (Iridaceae: Crocoideae) from eastern tropical Africa with notes on the morphology of the genus. S. Afr. J. Bot. 2015, 96, 37-41. [CrossRef]

34. DeBaggio, T.; Tucker, A.O. The Encyclopedia of Herbs: A Comprehensive Reference to Herbs of Flavor and Fragrance, 2nd ed.; Timber Press Inc.: Portland, OR, USA, 2009; pp. 266-267.

35. Troy, D.B. (Ed.) Remington: The Science and Practice of Pharmacy, 21st ed.; Lippincott William and Wilkins: Philadelphia, PA, USA, 2006; p. 1069.

36. Wang, H.; Cui, Y.; Zhao, C. Flavonoids of the genus Iris (Iridaceae). Mini Rev. Med. Chem. 2010, 10, 643-661. [CrossRef]

37. Iwashina, T.; Ootani, S. Flavonoids of the genus Iris: Structures, distribution and function. Ann. Tsukuba Bot. Gard. 1998, 17, 147-183.

38. Kukula-Koch, W.; Sieniawska, E.; Widelski, J.; Urjin, O.; Glowniak, P.; Skalicka-Woźniak, K. Major secondary metabolites of Iris spp. Phytochem. Rev. 2015, 14, 51-80. [CrossRef]

39. Roger, B.; Jeannot, V.; Fernandez, X.; Cerantola, S.; Chahboun, J. Characterisation and Quanti fi cation of Flavonoids in Iris germanica L. and Iris pallida Lam. Resinoids from Morocco. Phytochem. Anal. 2011, 23, 450-455. [CrossRef]

40. Mykhailenko, O. Composition of Volatile Oil of Iris pallida Lam. From Ukraine. Turk. J. Pharm. Sci. 2018, 15, 85-90. [CrossRef]

41. Board, N. Modern technology of perfumes, flavours and essential oils. Natl. Inst. Ind. Res. 2005, 2, $282-283$.

42. Fern, K.; Tropical Plants Database. Tropical.Theferns.Info. 2019. Available online: tropical.theferns.info/viewtropical.php?id= Harrisonia+perforata (accessed on 21 July 2021). 
43. Tuntiwachwuttikul, P.; Phansa, P.; Pootaeng-On, Y.; Taylor, W.C. Chromones from the branches of Harrisonia perforata. Chem. Pharm. Bull. 2006, 54, 44-47. [CrossRef] [PubMed]

44. Khuong-Huu, Q.; Chiaroni, A.; Riche, C.; Nguyen-Ngoc, H.; Nguyen-Viet, K.; Khuong-Huu, F. New rearranged limonoids from Harrisonia perforata. J. Nat. Prod. 2000, 63, 1015-1018. [CrossRef]

45. Khuong-Huu, Q.; Chiaroni, A.; Riche, C.; Nguyen-Ngoc, H.; Nguyen-Viet, K.; Khuong-Huu, F. New rearranged limonoids from Harrisonia perforata. III. J. Nat. Prod. 2001, 64, 634-637. [CrossRef] [PubMed]

46. Thu, H.D.T.; Tri, M.V.; Ngoc, N.B.; Sévenet, T.; Païs, M.; Martin, M.T. Foritin, a new limonoid from Harrisonia perforata. Nat. Prod. Lett. 2000, 14, 191-195. [CrossRef]

47. Yin, S.; Chen, X.; Su, Z.S.; Yang, S.P.; Fan, C.Q.; Ding, J.; Yue, J.M. Harrisotones A-E, five novel prenylated polyketides with a rare spirocyclic skeleton from Harrisonia perforata. Tetrahedron 2009, 65, 1147-1152. [CrossRef]

48. Choodej, S.; Sommit, D.; Pudhom, K. Rearranged limonoids and chromones from Harrisonia perforate and their anti-inflammatory activity. Bioorg. Med. Chem. Lett. 2013, 23, 3896-3900. [CrossRef] [PubMed] 